\title{
Asymptotic behaviour of solutions and their derivatives, for semilinear elliptic problems with blowup on the boundary
}

by

\author{
Catherine BANDLE \\ Mathematisches Institut, Universität Basel, Rheinsprung 21, \\ CH-4051 Basel, Switzerland. \\ and \\ Moshe MARCUS \\ Department of Mathematics, Technion I.I.T., 32000 Haifa, Israel.
}

\begin{abstract}
By means of comparison functions the asymptotic behaviour of solutions of semilinear elliptic equations which blow up at the boundary is established. The results depend only on the principal part of the second order operator and can be expressed in a simple way in terms of the associated Riemannian metric. In order to discuss the asymptotic behaviour of the derivatives a blowup technique together with a scaling argument is used.
\end{abstract}

Key words: Comparison principle, blowup techniques.

RÉsumÉ. - On utilise des fonctions de comparaison afin d'établir le comportement asymptotique des solutions de problèmes elliptiques semilinéaires qui explosent au bord. Les résultats s'expriment de manière

A.M.S. Classification: 35 J 65, 35 J 60.

Annales de l'Institut Henri Poincaré - Analyse non linéaire - 0294-1449 
simple à l'aide de la métrique de Riemann associée à cet opérateur. L'étude des dérivées repose sur une technique de blowup et utilise une invariance de groupe des solutions.

\section{INTRODUCTION}

Let $D \subset \mathbb{R}^{N}$ be a bounded domain and suppose that its boundary $\partial D$ is partitioned into two parts $\Gamma_{0}$ and $\Gamma$ where $\Gamma$ is relatively open with respect to $\partial D$ and satisfies an interior and exterior sphere condition.

In this paper we consider solutions to quasilinear problems of the form

$$
L u=g(x, u) \quad \text { in } \quad D, \quad u(x) \rightarrow \infty \quad \text { as } \quad x \rightarrow \Gamma
$$

where

$$
L=\sum_{i, j=1}^{N} a_{i j}(x) \frac{\partial^{2}}{\partial x_{i} \partial x_{j}}+\sum_{i=1}^{N} b_{i}(x) \frac{\partial}{\partial x_{i}}
$$

is a uniformly elliptic operator with $a_{i j}(x)=a_{j i}(x)$ and $a_{i j}, b_{i} \in C^{\alpha}(\bar{D})$.

We are interested in the precise behaviour of $u(x)$ as $x$ approaches $\Gamma$. The existence of such solutions under the assumptions listed below can be established by the method of upper and lower solutions together with (an extension of the uniform estimates of [4]. For an argument of this type see for instance [2]. The function $g(x, t)$ is supposed to grow sufficiently fast as $t \rightarrow \infty$ in the following sense.

$$
\lim _{t \rightarrow \infty} g(x, t) / f(t)=h(x) \quad \text { uniformly in } \bar{D}
$$

where $h(x)$ is an arbitrary continuous and positive function in $\bar{D}$ and $f(t)$ is subject to the conditions (F-1)-(F-3) listed below.

$$
\begin{aligned}
& f \in C^{1}[0, \infty) ; \quad f(t), f^{\prime}(t)>0 \text { for } t>t^{\prime} \geq 0 ; \\
& f \text { is unbounded }
\end{aligned}
$$

Denote by $F$ the primitive of $f$ such that $F\left(t_{0}\right)=0$ where, $t_{0}=\inf \{\tau \geq$ $t^{\prime}: f(t) \leq f(\tau)$ for every $\left.t \leq \tau\right\}$. Then

$$
\begin{aligned}
& \psi(t):=\int_{t}^{\infty}(2 F(s))^{-1 / 2}<\infty, \quad \forall t>t_{0} . \\
& \lim _{t \rightarrow \infty} \inf \psi(\beta t) / \psi(t)>1, \quad \forall \beta \in(0,1) .
\end{aligned}
$$


Typical examples are $f(t)=t^{p}, p>1$ and $f(t)=e^{t}$. Conditions (F-1) and (F-2) imply that $f$ is superlinear at infinity (cf. Appendix).

The special case $L=\Delta$ was treated in a series of papers ([4], [5], [6], [1], [2]). In particular it has been shown that

$$
u(x) / \phi(\sqrt{h(x)} \delta(x)) \rightarrow 1 \quad \text { as } \quad x \rightarrow \Gamma .
$$

Here $\phi(t)$ is the inverse of $\psi(t)$ and $\delta(x):=\operatorname{dist}(x, \partial D)$. It should be noted that $\phi$ is a solution to the 1 -dim. problem

$$
\phi^{\prime \prime}=f(\phi), \quad \phi(x) \rightarrow \infty \quad \text { as } \quad x \rightarrow 0 .
$$

In the first part we extend (1.2) to solutions of (1.1). It turns out that the asymptotic behaviour doesn't depend on the first order terms of $L$. The result is expressed in the most transparent way if we introduce in $D$ a Riemannian metric of the form

$$
d s^{2}=b_{i j}(x) d x_{i} d x_{j}
$$

where $b_{i j}(x)$ is the inverse matrix of $a_{i j}(x)$ and repeated indices are to be summed up from 1 to $N$. In this metric the distance between two points $P$ and $Q$ is given by $\inf _{\gamma} \int_{\gamma} d s, \gamma \subset D$ being an arc connecting $P$ and $Q$. Let $\hat{\delta}_{\Gamma}(x)$ be the distance from $x$ to $\Gamma$ in the metric (1.4). With this notation we can state the following result.

THEOREM 1.1. - The solutions of (1.1) satisfy (1.2) with $\delta(x)$ replaced by $\hat{\delta}_{\Gamma}(x)$.

In view of condition (F-1) this theorem holds also if $L$ is replaced by $L+\lambda(x)$ where $\lambda(x)$ is an arbitrary function in $C^{0}(\bar{D})$.

The result applies also to problems on Riemannian manifolds. If $L$ is the Laplace-Beltrami operator

$$
\Delta_{B}=\frac{1}{\sqrt{b}} \frac{\partial}{\partial x_{i}}\left(\sqrt{b} a_{i j}(x) \frac{\partial}{\partial x_{i}}\right), \quad b:=\operatorname{det}\left(b_{i j}\right)
$$

with respect to the metric $d s^{2}=b_{i j} d x_{i} d x_{j}\left[b_{i j}\right.$ is the inverse of $\left.a_{i j}\right]$, then $\hat{\delta}_{\Gamma}(x)$ corresponds to the distance in the metric $d s$.

If $\Gamma=\partial D, u$ is called a large solution of (1.1). A particular large solution is the maximal solution $U(x)$ characterized by

$$
U(x)=\sup \{u: L u=g(x, u) \text { in } D\} .
$$

This solution has been the object of many studies ([4], [5], [2]). It plays an important role in geometry [5] and probability theory [3]. If $g(x, t) / t$ is Vol. 12, $\mathrm{n}^{\circ}$ 2-1995. 
monotone increasing in $t$ for every fixed $x \in \bar{D}$, it turns out that it is the only solution such that $u(x) \rightarrow \infty$ as $x \rightarrow \partial D$ ([5], [2], [6]). This is a simple consequence of the asymptotic behaviour and the maximum principle.

Similar results were obtained by Véron [7] by a different method in the special case where $L$ contains only second order terms and $g=h(x) t^{p}$.

In the second part we derive the asymptotic behaviour of the derivatives of $u(x)$ near $\Gamma$ and extend results of [2]. In this part we shall assume that $a_{i j}$ is in $C^{1, \alpha}(\bar{D})$. Here we use a blowup technique introduced in [1] and a scaling argument. For this purpose we have to restrict (1.1) to the case where $g(x, t)$ satisfies (G-1) with $f=t^{p}, p>1$. If the main part of $L$ is the Laplacian and $h(x) \equiv 1$ we obtain,

$$
\begin{gathered}
|\nabla u|^{2} / \phi^{\prime 2}(\delta(x)) \rightarrow 1 \text { locally uniformly as } x \rightarrow \Gamma \text { with } \\
\phi(t)=\left(\frac{p-1}{\sqrt{2(p+1)}} t\right)^{-2 /(p-1)} .
\end{gathered}
$$

In the general case we get

$$
\frac{a_{i j}(x) \frac{\partial u}{\partial x_{i}}(x) \frac{\partial u}{\partial x_{j}}(x)}{\phi^{2}\left(\hat{\delta}_{\Gamma}(x)\right)} \rightarrow 1 \text { locally uniformly as } x \rightarrow \Gamma .
$$

Higher derivatives are also studied and it is shown that their asymptotic behaviour can be completely described in terms of $\phi$ and its derivatives only.

\section{ASYMPTOTIC BEHAVIOUR OF THE SOLUTIONS}

2.1. - Throughout this section we shall use the following notation. $A(x)$ is the symmetric matrix $\left(a_{i j}(x)\right)$ and $S(x)$ is an orthogonal matrix such that $S(x) A(x) S^{T}(x)=C(x)$ is of diagonal form. Since $L$ is uniformly elliptic, $A$ has an inverse and $C^{1 / 2}(x)$ and $C^{-1 / 2}(x)$ are well-defined. Let $(x, y)$ be the scalar product in $\mathbb{R}^{N}$ and $\langle x, y\rangle_{z}=\left(x, A^{-1}(z) y\right)$. Moreover put $\|x\|_{z}^{2}=\langle x, x\rangle_{z}$. Let $x$ be sufficiently near $\Gamma$ so that there is a unique $z \in \bar{\Gamma}$ such that $\|x-z\|_{z}=\inf _{z^{*} \in \Gamma}\left\|x-z^{*}\right\|_{z^{*}}$. Denote this infimum by $\delta_{\Gamma}(x)$ and the point $z$ by $z(x)$. Note that $\delta_{\Gamma}(x) / \hat{\delta}_{\Gamma}(x) \rightarrow 1$ as $x \rightarrow \Gamma$, although the two notions of distance do not coincide. If $\nu_{z}$ stands for the outer normal at $z$, then $z-x$ has the direction of $A(z) \nu_{z}$. Denote by $U$ a neighborhood of $\Gamma$ which has the property that for every $x \in U$ the point $z(x)$ is well-defined.

For every $x_{0} \in U \cap D$, let $E\left(x_{0}\right):=\left\{x:\left\|x-x_{0}\right\|_{z\left(x_{0}\right)}<\rho\left(x_{0}\right)\right\}$ be the largest ellipsoid contained in $D$. 
Let us introduce in $E\left(x_{0}\right)$ new coordinates

$$
y=C^{-1 / 2}\left(z\left(x_{0}\right)\right) S\left(z\left(x_{0}\right)\right)\left(x-x_{0}\right)=: T_{x_{0}}(x)
$$

$E\left(x_{0}\right)$ is then transformed into the ball $B\left(x_{0}\right):=\left\{|y|<\rho\left(x_{0}\right)\right\}$. By the chain rule

$$
\begin{gathered}
\frac{\partial}{\partial x_{i}}=\sigma_{k i} \frac{\partial}{\partial y_{k}}, \quad\left(\sigma_{i j}\right)=C^{-1 / 2}\left(z\left(x_{0}\right)\right) S\left(z\left(x_{0}\right)\right) \\
\frac{\partial^{2}}{\partial x_{i} \partial x_{j}}=\sigma_{\mu j} \sigma_{k i} \frac{\partial^{2}}{\partial y_{k} \partial y_{\mu}} .
\end{gathered}
$$

Putting $\hat{f}(y)=f(x(y))$ for any function $f$ in $E\left(x_{0}\right)$, we get

$$
L u(x)=L_{y} \hat{u}=\hat{a}_{i j} \sigma_{k i} \sigma_{\mu j} \frac{\partial^{2} \hat{u}}{\partial y_{k} \partial y_{\mu}}+\hat{b}_{i} \sigma_{k i} \frac{\partial \hat{u}}{\partial y_{k}} .
$$

Let $\varepsilon_{k l}(x)=\left[a_{i j}(x)-a_{i j}\left(z\left(x_{0}\right)\right)\right] \sigma_{k i} \sigma_{l j}$ and $d_{k}(y)=\hat{b}_{i}(y) \sigma_{k i}$.

Observe that

$$
\begin{aligned}
& \left(a_{i j}\left(z\left(x_{0}\right)\right) \sigma_{k i} \sigma_{l j}\right)= \\
& =C^{-1 / 2}\left(z\left(x_{0}\right)\right) S\left(z\left(x_{0}\right)\right) A\left(z\left(x_{0}\right)\right) S^{T}\left(z\left(x_{0}\right)\right) C^{-1 / 2}\left(z\left(x_{0}\right)\right)=\left(\delta_{k l}\right) .
\end{aligned}
$$

Thus if $u$ is a solution of (1.1) we have

$$
L_{y} \hat{u}=\Delta_{y} \hat{u}+\varepsilon_{k \mu} \frac{\partial^{2} \hat{u}}{\partial y_{k} \partial y_{\mu}}+d_{k} \frac{\partial \hat{u}}{\partial y_{k}}=g(x(y), \hat{u}) \text { in } B\left(x_{0}\right)
$$

for every $x_{0} \in U$.

Note that by (G-1), for any $\varepsilon>0$ there exists a number $t(\varepsilon)$ such that

$$
1-\varepsilon \leq g(x, t) / h(x) f(t) \leq 1+\varepsilon \quad \forall t \geq t(\varepsilon), \quad \forall x \in D
$$

Therefore by (F-1) and the maximum principle the following lemma holds.

LEMMA 2.1. - Let $\bar{u}$ (resp. $\underline{u}$ ) satisfy $L \bar{u} \leq(1-\varepsilon) h(x) f(\bar{u})$ or $L \underline{u} \geq$ $(1+\varepsilon) h(x) f(\underline{u})$ in a subdomain $D^{\prime} \subseteq D$. Suppose in addition that $\bar{u}$ (resp. $\underline{u}$ ) is bounded from below by $\max \left\{t_{0}, t(\varepsilon)\right\}$. Suppose that $u$ is a solution of equation (1.1) such that $u \geq \max \left\{t_{0}, t(\varepsilon)\right\}$. If $\bar{u} \geq u \quad(\underline{u} \leq u)$ on $\partial D^{\prime}$, then $\bar{u} \geq u \quad(\underline{u} \leq u)$ in $D^{\prime}$.

2.2. - Next we shall construct an upper bound for $u(x)$ near $\Gamma$. For every $\Gamma \subset \partial D$ and $\rho>0$ denote $\Gamma_{\rho}=\left\{x \in D: \delta_{\Gamma}(x)<\rho\right\}$.

Vol. $12, n^{\circ} 2-1995$. 
Let $\Gamma^{\prime}$ be a compact subset of $\Gamma$. Given $\varepsilon>0$, let $\rho(\varepsilon)>0$ be sufficiently small so that $\Gamma_{2 \rho(\varepsilon)}^{\prime} \subset U$ and

$$
u(x) \geq \max \left\{t_{0}, t(\varepsilon)\right\} \quad \forall x \in \Gamma_{\rho(\varepsilon)}^{\prime}
$$

and

$$
\begin{aligned}
& \left(1+\frac{1}{N}\right)\left|\varepsilon_{\kappa \mu} \frac{y_{\kappa} y_{\mu}}{|y|^{2}}\right|+\frac{1}{N}\left|\varepsilon \kappa \kappa+d_{\kappa} y_{\kappa}\right|<\varepsilon \\
& \forall y \in B\left(x_{0}\right) \text { and } \forall x_{0} \in \Gamma_{\rho(\varepsilon)}^{\prime} .
\end{aligned}
$$

The second inequality follows from the continuity of the coefficients of $L$.

Fix $x_{0} \in \Gamma_{\rho(\varepsilon)}^{\prime}$ and consider the problem

$$
\Delta V=\mu h_{*} f(V) \quad \text { in } \quad B=\left\{|y|<\rho\left(x_{0}\right)\right\}
$$

where $\mu=\frac{1-\varepsilon}{1+\varepsilon}$ and $h_{*}=\inf _{E\left(x_{0}\right)} h(x)$.

This problem possesses a maximal solution $V$ which is radially symmetric. By the maximum principle, $V$ is monotone increasing in $\left(0, \rho\left(x_{0}\right)\right)$. Therefore

$$
V^{\prime \prime} \leq \mu h_{*} f(V) \text { in }\left(0, \rho\left(x_{0}\right)\right) .
$$

Multiplication by $V^{\prime}$ and integration yields

$$
\int_{V(0)}^{\infty}[2 F(s)]^{-1 / 2} d s \leq \sqrt{\mu h_{*}} \rho .
$$

This estimate shows that $V(0) \rightarrow \infty$ as $\rho \rightarrow 0$.

In addition to the previous assumptions on $\rho(\varepsilon)$ we shall assume that it is sufficiently small so that

$$
V(0) \geq \max \left\{t_{0}, t(\varepsilon)\right\}
$$

where $V(r)$ is the maximal solution of (2.3) with $x_{0} \in \Gamma_{\rho(\varepsilon)}^{\prime}$. A straightforward computation yields $(r:=|y|)$

(2.4) $L_{y} V=\mu h_{*} f(V)+V^{\prime \prime} \varepsilon_{k l} y_{k} y_{l} / r^{2}+\frac{V^{\prime}}{r}\left[\varepsilon_{k k}-\varepsilon_{k l} y_{k} y_{l} / r^{2}+d_{k} y_{k}\right]$.

From (2.3), (iii) and (F-1) it follows that

$$
r^{N-1} V^{\prime}=\int_{0}^{r} s^{N-1} \mu h_{*} f(V) d s \leq \frac{\mu}{N} h_{*} r^{N} f(V(r))
$$


whence

$$
V^{\prime} / r \leq \frac{\mu}{N} h_{*} f(V)
$$

Furthermore we have

$$
V^{\prime \prime}=\mu h_{*} f(V)-(N-1) V^{\prime} / r \leq \mu h_{*} f(V)
$$

From (2.4)-(2.6) we get

$$
\begin{aligned}
L_{y} V \leq & \mu h_{*} f(V)\left[1+\left(1+\frac{1}{N}\right)\left|\varepsilon_{k l} \frac{y_{l} y_{k}}{r^{2}}\right|\right. \\
& \left.+\frac{1}{N}\left|\varepsilon_{k k}+d_{k} y_{k}\right|\right] .
\end{aligned}
$$

By (ii) and the definition of $\mu$

$$
L_{y} V \leq(1-\varepsilon) h_{*} f(V) \leq(1-\varepsilon) \hat{h} f(V) \text {. }
$$

By Lemma 2.1, this inequality together with (2.1) implies that $\hat{u}(y) \leq$ $V(y)$ in $B\left(x_{0}\right)$. (Here we use of course conditions (i) and (iii).) By (1.2)

$$
V(r) / \phi\left(\sqrt{\mu h_{*}}(\rho-r)\right) \rightarrow 1 \quad \text { as } \quad r \rightarrow \rho .
$$

Observe that for any $x$ lying on the segment $\overline{x_{0} z}$ with $z=z\left(x_{0}\right)$, $\rho-r=\|z-x\|_{z}$. Moreover

$$
\sqrt{\mu h_{*}}=\sqrt{h(z)}(1+o(1)) \quad \text { as } \quad x_{o} \rightarrow z
$$

From (2.9), we have for $x$ as above

$$
u(x) \leq\left(1+\eta_{1}\right) \phi\left(\sqrt{\left(1-\eta_{2}\right) h(z)}\|x-z\|_{z}\right)
$$

where $\eta_{1} \rightarrow 0$ as $x \rightarrow z$ and $\eta_{2} \rightarrow 0$ as $x_{0} \rightarrow z$ uniformly with respect to $x_{0}$ in $\Gamma_{\rho(\varepsilon)}^{\prime}$. By (F-3) we deduce (cf. Appendix, Lemma C) that for given $\varepsilon>0$ there exists $\delta(\varepsilon)$ such that

$$
u(x) \leq(1+\varepsilon) \phi\left(\sqrt{h(z)\|x-z\|_{z(x)}}\right) \quad \forall x \in \Gamma_{\delta(\varepsilon)}^{\prime} .
$$

2.3. - Next we shall construct a lower bound for $u(x)$ near $\Gamma$. Here we use a variant of a localization technique introduced in [6]. Denote by $E_{r}(\bar{x})$ the ellipsoid $\left\{\|x-\bar{x}\|_{z(\bar{x})}<r\right\}$. For $\bar{x} \in U \cap \bar{D}^{C}$, let $r_{0}(\bar{x})=\delta_{\Gamma}(\bar{x})$ and denote Vol. $12, \mathrm{n}^{\circ} 2-1995$. 
$E^{*}(\bar{x})=E_{r_{0}(\bar{x})}(\bar{x})$. For any $r_{1}>r_{0}(\bar{x})$, set $A_{r_{1}}(\bar{x})=E_{r_{1}}(\bar{x})-E^{*}(\bar{x})$. Let as before $\Gamma^{\prime}$ be a compact subset of $\Gamma$. In what follows we shall assume that $\bar{x}$ is such that $z(\bar{x}) \in \Gamma^{\prime}$ and that $r_{1}$ is sufficiently small so that $A_{r_{1}}(\bar{x}) \cap \partial D$ is a connected subset of $\Gamma^{\prime}$ and $D_{r_{1}}(\bar{x})=A_{r_{1}}(\bar{x}) \cap D$ is a connected subset of $D$.

Let $T_{\bar{x}} x$ be the linear transformation (*) defined in subsection 2.1. This transformation maps $A_{r_{1}}(\bar{x})$ into a spherical annulus

$$
\widehat{A}_{r_{1}}(\bar{x})=\left\{y: r_{0}(\bar{x})<\|y\|<r_{1}\right\}
$$

If $u$ is a solution of problem (1.1), then $\hat{u}$ satisfies $(2.1)$ in $\widehat{D}_{r_{1}}(\bar{x})=$ $T_{\bar{x}} D_{r_{1}}(\bar{x})$.

Given $\varepsilon>0$, there exists $\rho_{1}(\varepsilon)>r_{0}(\bar{x})$ such that if $r_{1} \in\left(r_{0}, \rho_{1}(\varepsilon)\right)$ then,

$$
u(x) \geq \max \left\{2 t_{0}, t(\varepsilon)\right\}, \quad \forall x \in D_{r_{1}}(\bar{x}),
$$

for every $\bar{x} \in U \cap \bar{D}^{C}$ such that $z(\bar{x}) \in \Gamma^{\prime}$.

Let $r_{1}$ be a number in this interval such that $r_{1}<2 r_{0}$. Denote by $v$ the radially symmetric solution of the problem

$$
\begin{aligned}
\Delta v & =\mu h^{*} f(v) \text { in } \widehat{A}_{r_{1}}(\bar{x}) \\
v\left(r_{1}\right) & =\inf _{D_{r_{1}}(\bar{x})} u, \quad v(r) \rightarrow \infty \text { as } r \rightarrow r_{0}(\bar{x})
\end{aligned}
$$

where $\mu=\frac{1+\varepsilon}{1-2 \varepsilon}$ and $h^{*}=\sup _{D_{r_{1}}(\bar{x})} h$. By the maximum principle, $v$ has no local maximum in $\left(r_{0}(\bar{x}), r_{1}\right)$. If $v$ has a local minimum at the point $r_{\text {min }}$ in this interval, we shall replace $r_{1}$ by the number $r_{1}^{\prime} \in\left(r_{0}(x), r_{\text {min }}\right)$ where $v\left(r_{1}^{\prime}\right)=v\left(r_{1}\right)$. Note that the value of $r_{1}^{\prime}$ depends only on $r_{0}(\bar{x})$ and $r_{1}$. Thus we may assume that $r_{1}$ has been so chosen that $v$ is monotone decreasing in $\left(r_{0}(\bar{x}), r_{1}\right)$. Since

$$
v^{\prime \prime}=-\frac{N-1}{r} v^{\prime}+\mu h^{*} f(v)
$$

it follows that $v^{\prime \prime}$ is positive and hence $v^{\prime}$ is monotone increasing. Consequently $v^{\prime 2}$ is monotone decreasing in $\left(r_{0}(\bar{x}), r_{1}\right)$. Thus

$$
\begin{aligned}
v^{\prime 2}\left(r_{1}\right)-v^{\prime 2}(r) & =-2(N-1) \int_{r}^{r_{1}} \frac{v^{\prime 2}(s)}{s} d s+2 \mu h^{*}\left[F\left(v\left(r_{1}\right)\right)-F(v(r))\right] \\
& \geq-2(N-1) v^{\prime 2}(r) \log \frac{r_{1}}{r}+2 \mu h^{*}\left[F\left(v\left(r_{1}\right)\right)-F(v(r))\right]
\end{aligned}
$$


Let $r_{1}$ be so close to $r_{0}(\bar{x})$ that $\log \frac{r_{1}}{r_{0}(\bar{x})} \leq \frac{1}{4(N-1)}$. Then

$$
v^{\prime 2}\left(r_{1}\right)-v^{\prime 2}(r) / 2 \geq 2 \mu h^{*}\left[F\left(v\left(r_{1}\right)\right)-F(v(r))\right] .
$$

Since $v\left(r_{1}\right) \geq \inf _{D_{\tau_{1}}(\bar{x})} u \geq 2 t_{0}$ it follows that

$$
F(v(r)) \geq F\left(v\left(r_{1}\right)\right) \geq F\left(2 t_{0}\right)>0 \quad \text { in } \quad\left(r_{0}(\bar{x}), r_{1}\right) .
$$

Therefore the previous inequality implies that there exists a positive number $c^{*}$ such that

$$
v^{\prime 2}(r) \leq c^{*} F(v(r)), \quad \forall r \in\left(r_{0}(\bar{x}), r_{1}\right) .
$$

(For instance we may put $\left.c^{*}=\left[4 \mu h^{*}+\frac{2 v^{\prime 2}\left(r_{1}\right)}{F\left(v\left(r_{1}\right)\right)}\right]\right)$. On the other hand our assumptions on $f$ imply that

$$
F^{1 / 2}(s) / f(s) \rightarrow 0 \quad \text { as } \quad s \rightarrow \infty .
$$

(See Lemma B of the Appendix). Consequently, for every $\varepsilon^{\prime}>0$ there exists $s\left(\varepsilon^{\prime}\right)$ such that

$$
\left|v^{\prime}(s)\right| \leq \varepsilon^{\prime} \mu h^{*} f(s), \quad \text { for } \quad s \geq s\left(\varepsilon^{\prime}\right) .
$$

From this inequality and (2.11) we obtain,

$$
\left|v^{\prime \prime}\right| \leq\left(1+\frac{N-1}{r_{0}(\bar{x})} \varepsilon^{\prime}\right) \mu h^{*} f(s) \text { for } s \geq s\left(\varepsilon^{\prime}\right) .
$$

Now $L_{y}$ can be computed as in (2.4) with $h_{*}$ and $x_{0}$ replaced by $h^{*}$ and $\bar{x}$ respectively. Assuming that $r_{1}$ is sufficiently small (so that the coefficients $\varepsilon_{k l}$ in (2.4) are small), and that $\varepsilon^{\prime}$ has been chosen sufficiently small we obtain from (2.12) and (2.13)

$$
L_{y} v \geq \mu h^{*}(1-2 \varepsilon) f(v), \text { whenever } v \geq s\left(\varepsilon^{\prime}\right) .
$$

The value of $\varepsilon^{\prime}$ is independent of the choice or $r_{1}$ (as long as $r_{1} \leq 2 r_{0}$ ). We shall assume that $r_{1}$ has been chosen sufficiently near to $r_{0}$ so that

$$
\inf _{D_{r_{1}}(\bar{x})} u \geq s\left(\varepsilon^{\prime}\right)
$$

Hence, by (2.14)

$$
L_{y} v \geq(1+\varepsilon) h^{*} f(v) \text { in } \quad \widehat{D}_{r_{1}}(\bar{x}) .
$$


Therefore, in view of the fact that $v \leq \hat{u}$ on the boundary of $\widehat{D}_{r_{1}}(\bar{x})$, Lemma 1.21 implies that $v \leq \hat{u}$ in $\widehat{D}_{r_{1}}(\bar{x})$. Now proceeding as in subsection 2.2 we conclude that

$$
u(x) \geq\left(1-\eta_{1}\right) \phi\left(\sqrt{\left(1+\eta_{2}\right) h(z)}\|x-z\|_{z}\right)
$$

for every $x$ in $D_{r_{1}}(\bar{x})$ which is colinear with $\bar{x}$ and $z=z(\bar{x})$. Using again Lemma $C$ of the Appendix we conclude that, for every $\varepsilon>0$ there exists $\delta(\varepsilon)$ such that

$$
u(x) \geq(1-\varepsilon) \phi\left(\sqrt{h(z)}\|x-z\|_{z}\right) \quad \forall x \in \Gamma_{\delta(\varepsilon)}^{\prime}
$$

where $z=z(x)$.

Combining estimates (2.10) and (2.15) we obtain

$$
u(x) / \phi\left(\sqrt{h(z)}\|x-z\|_{z}\right) \rightarrow 1 \text { as } x \rightarrow z, x \in D, z \in \Gamma,
$$

provided that $x-z$ is parallel to $A(z) \nu_{z}$. Furthermore the convergence is uniform with respect to $z$ in compact subsets of $\Gamma$. In the following theorem we restate this result in a form which does not explicitly involve the point $z$.

THEOREM 2.1. - Let $\Gamma^{\prime}$ be a compact subset of $\Gamma$. Then there exists a positive $\sigma$ such that

$$
u(x) / \phi\left(\sqrt{h(x)} \delta_{\Gamma}(x)\right) \rightarrow 1 \quad \text { as } \quad \delta_{\Gamma}(x) \rightarrow 0, x \in \Gamma_{\sigma}^{\prime} .
$$

\section{ASYMPTOTIC BEHAVIOUR OF THE DERIVATIVES}

In this section we study the asymptotic behaviour of the gradient and higher derivatives of solutions of problem (1.1), assuming that $g$ satisfies condition (G-1) with $f(t)=t^{p}$. First we shall discuss in detail the case

$$
g(x, t)=t^{p}, p>1 .
$$

After that we shall point out the modifications needed for the more general case. Our method combines the localization arguments of the previous sections with a blowup technique introduced in [1].

With the notation of section 2.1, let $x_{0}$ be a point in $U \cap D$ and set $y^{\prime}=T_{x_{0}}\left(z\left(x_{0}\right)\right)$. Let $\mathrm{O}$ denote the center of the ball $B\left(x_{0}\right)=\left\{|y|<\rho\left(x_{0}\right)\right\}$ and let $y_{0}$ be a point in the interior of the segment $\overline{\mathrm{O} y^{\prime}}$. Recall that $B\left(x_{0}\right)$ is a ball in the $y$-space where $y=T_{x_{0}}(x)$. Denote $\beta=\left|y_{0}-y^{\prime}\right|$, 
$\beta^{\prime}=\left|y_{0}-\mathrm{O}\right|=\rho\left(x_{0}\right)-\beta$. Let $\eta$ be a set of Cartesian coordinates centered at $y_{0}$ such that the positive $\eta_{1}$ axis is in the direction $\overline{y_{0} \mathrm{O}}$ and denote by $T_{x_{0}}^{\prime}$ the linear transformation $x \rightarrow \eta$. Choose a number $\delta \in(0,1 / 2)$ and denote,

$$
\begin{aligned}
D(\beta) & :=\left\{\eta:|\eta|<\beta^{1-\delta},|\eta-\mathrm{O}|<\beta^{\prime}\right\} \\
\gamma_{1, \beta} & :=\left\{\eta \in \partial D(\beta):|\eta-\mathrm{O}|=\beta^{\prime}\right\} \\
\gamma_{2, \beta} & :=\partial D(\beta) \backslash \gamma_{1}
\end{aligned}
$$

Clearly $\overline{D(\beta)}$ is contained in $B\left(x_{0}\right)$ for all $\beta \in\left(0, \rho\left(x_{0}\right)\right)$. Denote,

$$
\xi=\beta^{-1} \eta, \quad \widetilde{M}=\beta^{-1} M
$$

where $M$ is any set in $\mathbb{R}^{N}$. Let $\eta^{\prime}=\left(\eta_{2}, \ldots, \eta_{N}\right)$ and $\xi^{\prime}=\beta^{-1} \eta^{\prime}$. A straightforward computation yields,

$$
\begin{cases}0 \leq \eta_{1} \leq \beta^{2-2 \delta} / 2 \beta^{\prime}, & \forall \eta \in \gamma_{1, \beta} \\ \left|\eta^{\prime}\right| \leq \beta^{1}-\delta, & \forall \eta \in D(\beta)\end{cases}
$$

From this and the fact that $|\eta|=\beta^{1-\delta}$ on $\gamma_{2, \beta}$ we obtain,

$$
\begin{cases}\sup \left\{\xi_{1}: \xi \in \tilde{\gamma}_{1, \beta}\right\} \rightarrow 0 & \text { as } \quad \beta \rightarrow 0 \\ \widetilde{D}(\beta) \rightarrow\left\{\xi: \xi_{1}>0\right\} & \text { as } \quad \beta \rightarrow 0\end{cases}
$$

Let $u$ be a solution of (1.1). If $\tilde{u}$ denotes this function in terms of the $\eta$ coordinates in $B\left(x_{0}\right)$, then (2.1) can be rewritten in the form

$$
\Delta_{\eta} \tilde{u}+\varepsilon_{k . l}^{\prime} \frac{\partial^{2} \tilde{u}}{\partial \eta_{k} \partial \eta_{l}}+d_{k}^{\prime} \frac{\partial \tilde{u}}{\partial \eta_{k}}=\tilde{u}^{p}
$$

Let $\phi=\phi_{p}$ be defined as in section 2 with respect to $f(t)=t^{p}$ :

$$
\phi_{p}(s)=\left(\frac{p-1}{\sqrt{2(p+1)}} s\right)^{-2 /(p-1)} .
$$

\section{Setting}

$$
v_{\beta}(\xi)=\tilde{u}(\beta \xi) / \phi_{p}(\beta), \quad \forall \xi \in \widetilde{D}(\beta)
$$

we obtain

$$
\Delta_{\xi} v_{\beta}+\varepsilon_{k, l}^{\prime} \frac{\partial^{2} v_{\beta}}{\partial \xi_{k} \partial \xi_{l}}+\beta d_{k}^{\prime} \frac{\partial v_{\beta}}{\partial \xi_{k}}=\lambda v_{\beta}^{p}, \quad \forall \xi \in \widetilde{D}(\beta)
$$

where $\lambda=\beta^{2} \phi(\beta)^{p-1}=2(p+1) /(p-1)^{2}$. 
Now let $\Gamma^{\prime}$ be a compact subset of $\Gamma$. Given $\varepsilon_{0}>0$ choose $\sigma_{0}$ sufficiently small so that $\Gamma_{\sigma_{0}}^{\prime} \subset U$ and

$$
1-\varepsilon_{0} \leq u(x) / \phi_{p}\left(\delta_{\Gamma}(x)\right) \leq 1+\varepsilon_{0}, \quad \forall x \in \Gamma_{\sigma_{0}}^{\prime}
$$

(By Theorem 2.1 such a choice of $\sigma_{0}$ is possible.) Assume that $x_{0} \in \Gamma_{\sigma_{0}}^{\prime}$. Then if $\beta$ is sufficiently small,

$$
\beta \leq \delta_{\Gamma}(x(\eta)) \leq \beta+\beta^{1-\delta}, \quad \forall \eta \in D(\beta)
$$

where $x(\eta)=\left(T_{x_{0}}^{\prime}\right)^{-1}(\eta)$. Hence by $(3.8)$

$$
v_{\beta}(\xi) \leq 1+\varepsilon_{0}, \quad \forall \xi \in \widetilde{D}(\beta)
$$

for all sufficiently small $\beta$ (uniformly with respect to $x_{0}$ as above).

Next, let $\mu>0$ and consider a point $\eta \in D(\beta)$ such that $\eta_{1} \leq \mu \beta$. Then

$$
\beta \leq \delta_{\Gamma}(x(\eta)) \leq(1+\mu) \beta+\mathrm{O}\left(\left|\eta^{\prime}\right|^{2}\right)
$$

and by (3.3),

$$
1 \leq \delta_{\Gamma}(x(\eta)) / \beta \leq 1+\mu+\mathrm{O}\left(\beta^{1-2 \delta}\right) .
$$

By (3.9) $\sup _{D(\beta)} \delta_{\Gamma}(x(\eta)) \rightarrow 0$ as $\beta \rightarrow 0$. Hence by Theorem 2.1,

$$
\tilde{u}(\eta) / \phi_{p}\left(\delta_{\Gamma}(x(\eta))\right)=1+\mathrm{o}_{\beta}(1), \forall \eta \in D(\beta),
$$

where $o_{\beta}(1)$ is a quantity which tends to zero as $\beta \rightarrow 0$, uniformly with respect to $\eta$ in $D(\beta)$. From (3.11) and (3.12) we obtain,

$$
\begin{aligned}
& \left(1+o_{\beta}(1)\right)\left(1+o_{\beta}(1)+\mu\right)^{-2 /(p-1)} \leq \frac{\tilde{u}(\eta)}{\phi(\beta)} \\
& \leq 1+o_{\beta}(1) \quad \text { in }\left\{\eta \in D(\beta): \eta_{1} \leq \mu \beta\right\}
\end{aligned}
$$

Therefore, for every $\varepsilon>0$ there exist positive numbers $\beta_{\varepsilon}$ and $\mu_{\varepsilon}$ such that for every $\beta \in\left(0, \beta_{\varepsilon}\right)$,

$$
\left|v_{\beta}(\xi)-1\right| \leq \varepsilon \quad \text { in }\left\{\xi \in \widetilde{D}(\beta): \xi_{1} \leq \mu_{\varepsilon}\right\} .
$$

Further, by (3.3),

$$
\delta_{\Gamma}(x(\eta)) \leq \beta+|\eta|^{2} \leq \beta+\mathrm{O}\left(\beta^{2-2 \delta}\right) \quad \forall \eta \in \gamma_{1, \beta} .
$$


Therefore, by (3.11) and (3.13),

$$
\lim _{\beta \rightarrow 0} v_{\beta}(\xi)=1 \quad \text { uniformly for } \xi \in \tilde{\gamma}_{1, \beta} \text {. }
$$

Next let $\mu>0$ and consider a point $\eta$ in $D(\beta)$ such that $\eta_{1}>\mu \beta$. For such a point,

$$
\begin{aligned}
\delta_{\Gamma}(x(\eta)) & \geq(\mu+1) \beta+\mathrm{O}\left(\left|\eta^{\prime}\right|\right)^{2} \\
& \geq(\mu+1) \beta+\mathrm{O}\left(\beta^{2-2 \delta}\right) .
\end{aligned}
$$

Hence, for sufficiently small $\beta$,

$$
\delta_{\Gamma}(x(\eta)) / \beta \geq \mu
$$

and consequently, by (3.12),

$$
\frac{\tilde{u}(\eta)}{\phi_{p}(\beta)} \leq\left(1+\mathrm{O}_{\beta}(1)\right) \mu^{-2 /(p-1)} .
$$

Therefore, for every $\varepsilon>0$ there exists $\mu_{\varepsilon}^{\prime}$ such that

$$
v_{\beta}(\xi) \leq \varepsilon \quad \text { in }\left\{\xi \in \widetilde{D}(\beta): \xi_{1} \geq \mu_{\varepsilon}^{\prime}\right\}
$$

for all sufficiently small $\beta$ (independent of $\varepsilon$ ).

Let $K$ be a compact subset of the upper half space $\left\{\xi_{1}>0\right\}$. For all sufficiently small positive $\beta, K \subset \widetilde{D}(\beta)$ and (by (3.10)) $v_{\beta}$ is bounded by a bound independent of $\beta$. Therefore as $v_{\beta}$ satisfies (3.7), local estimates for solutions of linear elliptic equations imply that, for every $\alpha \in(0,1), v_{\beta}$ is bounded in $C^{2, \alpha}(K)$ by a bound independent of $\beta$, for all sufficiently small $\beta$. Hence there exists a sequence $\beta_{n} \rightarrow 0$ such that $\left\{v_{\beta_{n}}\right\}$ converges locally in $C^{2}$ in the upper half space $\xi_{1}>0$ to a function $v^{*}$ which satisfies,

$$
\Delta v^{*}=\lambda v^{*^{p}} \quad \text { in }\left\{\xi_{1}>0\right\} \text {. }
$$

Here we use the fact that (by (3.3)) $D(\beta)$ is contained in a ball $\left|y-y^{\prime}\right|<r(\beta)$ with $r(\beta) \rightarrow 0$ as $\beta \rightarrow 0$. Consequently

$$
\sup _{D(\beta)}\left|\varepsilon_{k, l}\right| \rightarrow 0 \quad \text { as } \beta \rightarrow 0,
$$

and of course the same statement holds w.r. to $\varepsilon_{k, l}^{\prime}$. Further, by (3.13) and (3.15),

$$
\begin{cases}v^{*}(\xi) \rightarrow 1 & \text { as } \xi_{1} \rightarrow 0 \\ v^{*}(\xi) \rightarrow 0 & \text { as } \xi_{1} \rightarrow \infty\end{cases}
$$


It is readily seen that problem (3.16), (3.17) possesses a unique solution, namely,

$$
v^{*}(\xi)=\left(1+\xi_{1}\right)^{-2 /(p-1)} .
$$

Therefore it follows that

$$
\lim _{\beta \rightarrow 0} v_{\beta}=v^{*}
$$

locally in $C^{2}\left(\left\{\xi_{1}>0\right\}\right)$. By repeated differentiation of (3.7) it also follows that $v_{\beta} \rightarrow v^{*}$ locally in $C^{m}\left(\left\{\xi_{1}>0\right\}\right)$ provided that the coefficients of $L$ are in $C^{m-1, \alpha}(\vec{D})$.

Consider a point $\eta=(\mu \beta, 0, \ldots, 0)$ where $\mu$ is a fixed positive number. Then, for $\beta \in\left(0, \rho\left(x_{0}\right)\right)$,

$$
\delta_{\Gamma}(x(\eta))=(1+\mu) \beta
$$

If $\xi=(\mu, 0, \ldots, 0)$ then, as $\beta \rightarrow 0$,

$$
\frac{\partial v_{\beta}}{\partial \xi_{k}}(\xi) \rightarrow \frac{\partial v^{*}}{\partial \xi_{k}}(\xi)= \begin{cases}-\frac{2}{p-1}(1+\mu)^{-\frac{p+1}{p-1}}, & \text { if } k=1 \\ 0, & \text { otherwise }\end{cases}
$$

so that

$$
\frac{1}{\phi_{p}^{\prime}(\beta)} \frac{\partial \tilde{u}(\eta)}{\partial \eta_{k}} \rightarrow \begin{cases}(1+\mu)^{-\frac{p+1}{p-1}}, & \text { if } k=1 \\ 0, & \text { otherwise. }\end{cases}
$$

Hence, by (3.20),

$$
\left|\nabla_{\eta} \tilde{u}(\eta)\right| / \phi_{p}^{\prime}\left(\delta_{\Gamma}(x(\eta))\right) \rightarrow 1 \text { as } \beta \rightarrow 0
$$

Since $\left|\nabla_{\eta} \tilde{u}\right|=\left|\nabla_{y} \hat{u}\right|,(3.22)$ implies,

$$
(A \nabla u(x), \nabla u(x))^{1 / 2} / \phi_{p}^{\prime}\left(\delta_{\Gamma}(x)\right) \rightarrow 1 \quad \text { as } \quad \beta \rightarrow 0
$$

for $x=x(\eta)$ with $\eta=(\mu \beta, 0, \ldots, 0)$. But, for sufficiently small $\sigma$ any point $x \in \Gamma_{\sigma}^{\prime}$ can be written in the above form with respect to an appropriately chosen $x_{0}$ in $\Gamma_{\sigma_{0}}^{\prime}$. Indeed $x_{0}$ can be any point in $\Gamma_{\sigma_{0}}^{\prime}$ such that $z(x)=z\left(x_{0}\right)$ and $x$ lies on the segment $\overline{z\left(x_{0}\right), x_{0}}$. Furthermore the convergence is uniform with respect to $x_{0}$ in $\Gamma_{\sigma_{0}}^{\prime}$. Thus (3.23) holds uniformly with respect to $x_{0} \in \Gamma_{\sigma_{0}}^{\prime}$. 
We also note that the argument is local involving only values of $u$ in a small neighborhood of $\Gamma^{\prime}$. Therefore if we consider equation (1.1) with a more general function, only the behaviour of $g$ for large values of $t$ is relevant. Consequently (3.23), appropriately modified, remains valid for $g$ satisfying (G-1) with $f(t)=t^{p}$. Thus we obtain,

TheOREM 3.1. - Assume that $g$ satisfies (G-1) with $f(t)=t^{p}$. Let $\Gamma^{\prime}$ be a compact subset of $\Gamma$ and let $\Gamma_{\sigma}^{\prime}$ denote a $\sigma$-neighborhood of $\Gamma^{\prime}$. If $\sigma$ is sufficiently small then, for $x \in \Gamma_{\sigma}^{\prime}$,

$$
(A \nabla u, \nabla u)^{1 / 2} / \sqrt{h(x)} \phi_{p}^{\prime}\left(\sqrt{h(x)} \delta_{\Gamma}(x)\right) \rightarrow 1
$$

as $\delta_{\Gamma}(x) \rightarrow 0$.

Remarks. - (1) From (3.21a) we conclude that

$$
\frac{\partial u}{\partial t}(x) \rightarrow 0 \quad \text { as } \quad x \rightarrow \Gamma
$$

for any tangential direction $t$ at $z(x)$. As before the convergence holds uniformly with respect to $x \in \Gamma_{\sigma_{0}}^{\prime}$.

(2) The arguments apply also to higher order derivatives of $u$ if they exist. For the special case

$$
L=\Delta+\sum_{i=1}^{N} b_{i} \frac{\partial}{\partial x_{i}}
$$

we get

$$
\frac{\partial^{k} u}{\partial n^{k}}(x) / h^{k / 2}(x) \phi_{p}^{(k)}\left(\sqrt{h(x)} \delta_{\Gamma}(x)\right) \rightarrow 1 \quad \text { as } x \rightarrow \Gamma .
$$

Here $n=\nu_{z}$ is the outer normal at $z(x)$ and $\delta_{\Gamma}(x)$ is measured in the Euclidean metric.

In the general case $n$ has to be replaced by $A(z) \nu_{z}=: \tilde{n}$ (cf. Section 2.1) and the left hand side has to be multiplied by $\left(\|\tilde{n}\|_{z} /|n|\right)^{k}$.

\section{APPENDIX}

Lemma $\mathrm{A}^{(1)}$. - Let $f(t)$ satisfy (F-1) and (F-2). Then

$$
\lim _{t \rightarrow \infty} F(t) / t^{2}=\infty \text { where } F(t)=\int_{t_{0}}^{t} f(s) d s .
$$

( ${ }^{1}$ We are indebted to Prof. M. Essèn for this lemma.

Vol. $12, \mathrm{n}^{\circ} 2-1995$. 
Proof. - By (F-1), $F(t)$ is convex for $t \geq t_{0}$. Suppose that

$$
\lim _{t \rightarrow \infty} \inf F(t) / t^{2}=\nu<\infty
$$

Since $\psi$ exists we must have $\varlimsup_{t \rightarrow \infty} F(t) / t^{2}=\infty$. Hence there is a sequence $t_{k} \rightarrow \infty$ as $k \rightarrow \infty$ such that for $b=\nu+1, F\left(t_{k}\right)=b t_{k}^{2}$. By the convexity we have

$$
F(t) \leq b t_{k}^{2}+t b \frac{t_{k+1}^{2}-t_{k}^{2}}{t_{k+1}-t_{k}}=: b L(t) \text { in }\left[t_{k}, t_{k+1}\right] .
$$

Observe that

$$
\int_{t_{k}}^{t_{k+1}} L^{-1 / 2}(t) d t=2 \frac{t_{k+1}-t_{k}}{t_{k+1}+t_{k}} \leq \sqrt{b} \int_{t_{k}}^{t_{k+1}} F^{-1 / 2}(t) d t .
$$

Since $\psi(t)<\infty$, the series $\sum_{k \geq k_{0}} \frac{t_{k+1}-t_{k}}{t_{k+1}+t_{k}}$ converges. Thus for $k$ sufficiently large, $\frac{t_{k+1}-t_{k}}{t_{k+1}+t_{k}}<1 / 2$ and $t_{k+1} \leq 3 t_{k}$.

But then $\left(t_{k+1}-t_{k}\right) /\left(t_{k+1}+t_{k}\right) \geq\left(t_{k+1}-t_{k}\right) / 4 t_{k}$ and

$$
\infty>\sum_{k \geq k_{0}} \frac{t_{k+1}-t_{k}}{4 t_{k}} \geq \int_{k_{0}}^{\infty} \frac{d t}{4 t}=\infty
$$

This contradiction shows that (1) is false. The proof is thus completed. In view of $(\mathrm{F}-1)$ we have

$$
F(t)-F\left(t_{0}\right) \leq f(t)\left(t-t_{0}\right), \forall t \geq t_{0}
$$

This together with Lemma A yields,

Lemma B. - Assume (F-1) and (F-2). Then

$$
\lim _{t \rightarrow \infty} f(t) / t=\infty
$$

From the monotonicity of $\phi$ we infer that for $\eta>0$ and $\delta \leq \delta_{0}$

$$
\phi((1-\eta) \delta) \geq \phi(\delta)
$$

(F-3) implies a partially converse inequality. 
Lemma C. - Let $\psi \in C\left[t_{0}, \infty\right)$. Suppose that $\psi$ is strictly monotone decreasing and satisfies (F-3). Let $\phi:=\psi^{-1}$. Then for every $\gamma>1$ there exist positive numbers $\eta_{\gamma}, \delta_{\gamma}$ such that,

$$
\phi((1-\eta) \delta) \leq \gamma \phi(\delta), \quad \forall \eta \in\left[0, \eta_{\gamma}\right], \quad \forall \delta \in\left[0, \delta_{\gamma}\right]
$$

The proof is straightforward and will be omitted.

\section{REFERENCES}

[1] C. BANDLE and M. Essèn, On the Solutions of Quasilinear Elliptic problems with Boundary Blow-up, Symposia Matematica, Vol. 35, 1994, pp. 93-111.

[2] C. Bandle C. and M. Marcus M., Large solutions of semilinear elliptic equations: existence, uniqueness and asymptotic behaviour, $J$. d'Anal. Mathém., Vol. 58, 1992, pp. 9-24.

[3] E. B. DYNkIN, A probabilistic approach to one class of nonlinear differential equations, Probab. Theory Rel. Fields, Vol, 90, 1991, pp. 89-115.

[4] J. B. Keller, On solutions of $\Delta u=f(u)$, Comm. Pure Appl. Math., Vol. 10, 1957, pp. 503-510.

[5] C. LOEWner and L. Nirenberg, Partial differential invariant under conformal or projective transformations, Contributions to Analysis (L. Ahlfors ed.), Acad. Press N. Y., I974, pp. 245-272.

[6] M. MARCus, On solutions with blow-up at the boundary for a class of semilinear elliptic equations, Developments in Partial Differential Equations and Applications (Buttazzo et al ed.), Plenum Press, 1992, pp. 65-79.

[7] L. VÉron, Semilinear elliptic equations with uniform blow-up on the boundary, J. d' Anal. Mathém., Vol. 58, 1992. 